Z Epileptol 2019 $\cdot 32: 4-5$

https://doi.org/10.1007/s10309-018-0240-8

(c) Springer Medizin Verlag GmbH, ein Teil von Springer Nature 2019

CrossMark

\section{Berthold Voges ${ }^{1} \cdot$ Ramin Khatami ${ }^{2,3}$}

'Epilepsiezentrum Hamburg, Evangelisches Krankenhaus Alsterdorf, Hamburg, Deutschland

${ }^{2}$ Zentrum für Schlafmedizin, Schlafforschung und Epileptologie, Klinik Barmelweid AG, Barmelweid, Schweiz

${ }^{3}$ Neurologische Klinik für Neurologie, Inselspital, Universitätsspital Bern, Universität Bern, Bern, Schweiz

\title{
Epilepsie und Schlaf - mehr als Tag und Nacht
}

The greatest obstacle to discovery is not ignorance but the illusion of knowledge. (Daniel Boorstin, Direktor der Library of Congress, Washington, USA, 1978)

In der interdisziplinären Schnittmenge von Epileptologie und Schlafmedizin haben wir keinen Anlass, uns der Illusion hinzugeben, dass wir viel wüssten. Daher sind wir in der glücklichen Lage, ohne Hürden Neuentdeckungen machen und für beide Fachgebiete zusammenbringen zu können. Hierfür stehen uns neue Methoden und Techniken zur Verfügung, wie z.B. invasives EEG, High-DensityLangzeit-EEG-Analysen, Single Photon Computed Tomogtaphy (SPECT), Wearables mit weiter Verbreitung auch im nichtmedizinischen Einsatz sowie die zunehmende, auch somnologische Aufarbeitung epileptologischer Patienten im Langzeit-EEG-Monitoring.

Im Licht dieser neuen Erkenntnisse können sowohl Epilepsien wie auch schlafmedizinische Erkrankungen, z. B. Narkolepsie, Schlafapnoesyndrom, NREM-Parasomnien, REM-Schlafverhaltensstörung oder Schlafphasenstörungen, besser verstanden und daher besser behandelt werden, dies auch unter Berücksichtigung ihrer gegenseitigen Interaktionen.

Für die aktuelle Ausgabe der ZEPI konnten wir Autoren gewinnen, die mit großem Engagement in den Schnittstellen von Somnologie und Epileptologie arbeiten, über den aktuellen Kenntnisstand in ihren Bereichen zu berichten wissen und dabei eigene Erfahrungen einfließen lassen.
Das Heft beginnt mit Beiträgen zu Differenzialdiagnosen im Grenzbereich zur Epilepsie. Den Anfang macht Herr Carlos Schenck, Minneapolis, mit einer Übersicht über das nach ihm benannte Syndrom bzw. die „REM-behavior-disorder" (RBD): Typische und weniger typische klinische Manifestationen werden vorgestellt, die Pathophysiologie diskutiert, dies auch anhand von Tiermodellen. Elektrophysiologische Befunde werden besprochen, auch zur Abgrenzung gegen Differenzialdiagnosen und häufige Komorbiditäten, und schließlich wird die symptomatische RBD mit ihren behandelbaren oder vermeidbaren Auslösern differenziert von der mit Synucleinopathie assoziierten, idiopathischen RBD.

Im folgenden Artikel widmen sich Herr Ramin Khatami, Barmelweid, und Herr Ulf Kallweit, Hagen, der Narkolepsie und legen den klinischen Fokus auf Kataplexie und automatische Handlungen unter Berücksichtigung der Differenzialdiagnose $\mathrm{zu}$ epileptischen Anfällen. Zudem werden neue pathogenetische Daten vorgestellt, die eine (Auto-)Immungenese der Narkolepsie stützen.

Daran anschließend findet sich eine Übersichtsarbeit von Berthold Voges, Hamburg, über Parasomnien und deren Abgrenzung von schlafgebundenen epileptischen Anfällen. Als Leiter sowohl einer großen Epilepsie-Monitoring-Unit (EMU) als auch eines in das EMU integrierten neurologischen Schlaflabors kann er über spannende Einblicke in dieses Grenzgebiet zwischen Somnologie und Epileptologie berichten, dies erst anhand exemplarischer Fallbeispiele und dann systematisiert mit zusammenfassender Darstellung der Unterschiede in den jeweiligen Symptomkonstellationen. Auf gerne genutzte Fallstricke bei schwierigen Differenzialdiagnosen wird eingegangen. Mit Bezug auf die zahlreichen klinischen Überschneidungen zwischen NREM-Parasomnien und schlafgebundenen Anfällen werden aktuelle neurophysiogische Erkenntnisse und Hypothesen zur Pathophysiologie beider Syndrome erläutert und damit auch die Ähnlichkeiten in der Manifestation dieser Krankheiten begründet.

J. Rémi, München, und Ramin Khatami stellen in ihrem gemeinsamen Beitrag zum invasiven EEG neue Befunde vor, die innovative Einblicke sowohl in die Systemphysiologie des Schlafs wie auch in die Epileptologie ermöglichen. Die zunehmende Nutzung von invasivem EEG-Monitoring hat unsere Konzepte von globalen Schlaf- und Wachzuständen grundlegend verändert und auf den Kopf gestellt.

Die Bedeutung der Chronobiologie für Lebensqualität, Gesundheit und Krankheit im Allgemeinen wurde lange (und wird immer noch) massiv unterschätzt. Gerade im Bereich der Epileptologie spielen chronobiologische Rhythmen eine große Rolle. Darauf geht Peter Young, Münster, in seinem Artikel Chronobiologie des Schlafes und dessen Einfluss auf Epilepsien ein.

In Deutschland leben ca. 800.000 Menschen mit Epilepsie und nehmen Tag für Tag (und Nacht für Nacht) antikonvulsive Medikamente ein. Es ist kaum zu verstehen, warum bei dieser großen Gruppe an Betroffenen derart 
wenig bekannt ist über die Auswirkung der Antikonvulsiva auf Schlafqualität und Schlafstruktur und dadurch eben auch auf Vigilanz, kognitive Performance sowie Lebensqualität tagsüber. Auch besteht die Gefahr, durch medikamenteninduzierte Schlaffragmentierung den antikonvulsiven Effekt der Medikamente zu konterkarieren. Qualitativ hochwertige Studien zu diesem Thema gibt es kaum, ebenso wenig wie Arbeiten $\mathrm{zu}$ objektiven Schlafparametern unter Antikonvulsiva. Frau Anna Heidbreder hat die große Aufgabe übernommen, etwas Licht in dieses Dunkel zu werfen und aus den vorliegenden Erkenntnissen Empfehlungen abzuleiten für eine rationale Auswahl adäquater Antikonvulsiva für Patienten mit Schlafstörungen.

Das obstruktive Schlafapnoesyndrom (OSAS) als häufigste schlafbezogene Atemstörungen ist mit $10 \%$ Prävalenz bereits in der Allgemeinpopulation eine weitverbreitete Krankheit. Bei Patienten mit therapieschwieriger Epilepsie ist sie mit $25 \%$ noch wesentlich häufiger anzutreffen und damit eine wesentliche Ursache für Tagesschläfrigkeit, aber auch für Hypertonie, Herzinsuffizienz, Diabetes, kardio- und zerebrovaskuläre Ereignisse. Darüber hinaus stellt ein unbehandeltes OSAS eine angehbare (!) Ursache für ein Nichtansprechen auf antikonvulsive Therapien dar. Herr Michal Lanz, Hamburg, zeigt auf, wie Tagesschläfrigkeit als eine Folge von z. B. antikonvulsiver Medikation unterschieden werden kann von Hypersomnie als Ausdruck schlafbezogener Atemstörungen; er referiert die aktuelle Literatur zur bidirektionalen Interaktion von OSAS einerseits und Epilepsie, Anfallspersistenz bzw. antikonvulsiven Therapien andererseits. Fallbeispiele aus der klinischen Praxis illustrieren die Bedeutung von korrekter Diagnose und - ggf. adäquater Behandlung von schlafbezogenen Atemstörungen und anderen Arousal-induzierenden Faktoren für eine erfolgreiche Epilepsietherapie.

Abschließend widmen sich Herr Max Christian Pensel und Herr Rainer Surges, beide Aachen, der dramatischen Problematik des SUDEP (,sudden unexpected death in epilepsy"), der den Patient nach Anfällen, üblicherweise im Schlaf, ereilen kann. Die bisher bekannten pathophysiologischen Faktoren werden besprochen; aufbauend darauf werden mögliche Präventions- und Gegenmaßnahmen skizziert wie Möglichkeiten zur frühen Wiederherstellung von Atmungs- und Kreislauffunktion im akuten Fall, aber auch die präventive Bedeutung maximal möglicher Anfallskontrolle durch Antiepileptika, Epilepsiechirurgie und epileptologische Stimulationstherapien.

Wir bedanken uns sehr herzlich bei den Autoren für die sehr gelungenen Beiträge, wünschen dem Leser einige neue Erkenntnisse, aber auch viel Vergnügen bei der Lektüre und sind überzeugt, dass Sie von den hier dargestellten Inhalten in Ihrem täglichen klinischen Schaffen unterstützt werden können.

Ein großer Dank geht auch an den Springer Verlag, namentlich an Frau Petra Elster und an Frau Petra Meyervom Hagen, für viel Geduld und tatkräftige Unterstützung bei der Realisierung dieser Ausgabe, und schließlich an die beiden Herausgeber dieser Zeitschrift, Herrn Adam Strzelczyk und Herrn Friedhelm Schmitt, für die Einladung an uns, ein ganzes ZEPI-Heft zu diesem bedeutsamen Thema gestalten zu dürfen.

Ramin Khatami, Barmelweid

Berthold Voges, Hamburg

\section{Korrespondenzadresse}

Dr. Berthold Voges
Epilepsiezentrum Hamburg,
Evangelisches Krankenhaus
Alsterdorf
Elisabeth-Flügge-Str. 1,
22337 Hamburg, Deutschland
b.voges@alsterdorf.de

Interessenkonflikt. B. Voges und R. Khatami geben an, dass kein Interessenkonflikt besteht. 